ISSN 2179-6750

\title{
Aplicabilidade da Teoria do Déficit do Autocuidado de Orem no Brasil: uma revisão integrativa
}

Applicability of the deficit theory of Orem's self-care in Brazil: an integrative review

Aplicabilidad de la Teoría de Déficit de Autocuidado de Orem: una revisión integradora

\author{
Maria Luiza Rêgo BEZERRA ${ }^{(1)}$ \\ Renata de Paula Rocha FARIA ${ }^{(1)}$ \\ Cristine Alves Costa de JESUS ${ }^{(1)}$ \\ Paula Elaine Diniz dos REIS ${ }^{(1)}$ \\ Diana Lúcia Moura PINHO ${ }^{(1)}$ \\ Ivone $\operatorname{KAMADA}^{(1)}$
}

Recebido: 18 maio 2017

Revisado: 10 jan 2018

Aceito: 14 jan 2018

Autor de correspondência: Maria Luiza Rêgo Bezerra marialuizaregobezerra@gmail.com

Conflito de interesses: Os autores declaram não haver nenhum interesse profissional ou pessoal que possa gerar conflito de interesses em relação a este manuscrito.
(1) Universidade de Brasília - UnB, Faculdade de Ciências da Saúde, Programa de Pós-Graduação em Enfermagem, Brasília, DF, Brasil.

\section{Resumo}

Objetivo: descrever o cenário da aplicabilidade da Teoria do Déficit de Autocuidado de Orem no âmbito da enfermagem brasileira. Método: revisão integrativa de 12 produções científicas compreendidas entre 2009-2014 a partir de critérios de inclusão e exclusão e dos descritores em saúde Teorias de Enfermagem", "Autocuidado" e "Cuidados de Enfermagem". Resultados: Observou-se no âmbito nacional maior produtividade sobre a Teoria do Déficit do Autocuidado de Orem em 2013 e tipologia mais ocorrente, estudos descritivos quantitativos e qualitativos, de igual ocorrência. A partir da análise das produções evidenciadas emergiram três categorias: "Sistematização da Assistência de Enfermagem (SAE)", "Aplicabilidade da Teoria" e "Educação em saúde". Conclusões: A Teoria do Déficit do Autocuidado de Orem, bem como seus postulados, ou seja, suas três teorias inter-relacionadas: Teoria do Autocuidado, Teoria do Déficit do Autocuidado e Teoria de Sistemas, abrangem inúmeros conceitos, de natureza epistemológica, que podem ser aplicados em diferentes grupos e situações.

Descritores: Teorias de Enfermagem; Autocuidado; Cuidados de Enfermagem. 


\begin{abstract}
Objective: to describe the scene of the applicability of the Self-care Deficit Theory within the brazilian nursing. Method: an integrative review of 12 scientific publications between 2009-2014 from inclusion and exclusion and health descriptors criteria "Nursing Theory", "Self-Care" and" Nursing Care". Results: It was observed at the national level higher productivity on the Self-care Deficit Theory in 2013 and more frequently occurring type, quantitative and qualitative descriptive studies, the same occurrence. From the analysis of the evidenced productions three categories emerged: "Systematization of Nursing Assistance (SAE)", "Theory of Applicability" and "Health education". Conclusions: The Self-care Deficit Theory and its postulates, namely its three interrelated theories: Self-Care Theory, Deficit Theory of Self-Care and Systems Theory, covering numerous concepts, epistemological, which can be applied in different groups and situations.
\end{abstract}

Keywords: Nursing Theory; Self-care; Nursing care.

\title{
Resumen
}

Objetive: describir la escena de la aplicabilidad de la teoría del déficit de autocuidado de Orem bajo la enfermería brasileña. Método: una revisión integradora de 12 publicaciones científicas entre 20092014 de inclusión y exclusión y de salud descriptores Teorías criterios de enfermería "," cuidado de sí mismo "y" Cuidados de enfermería". Resultados: Se observó a nivel nacional una mayor productividad en la Teoría del Déficit de Autocuidado de Orem en 2013 y que ocurren con mayor frecuencia tipo, estudios descriptivos cuantitativos y cualitativos, la misma ocurrencia. A partir del análisis de las producciones destacó surgieron tres categorías: "Sistematización de la Asistencia de Enfermería (SAE)", "Teoría de la aplicabilidad" y "Educación para la salud". Conclusiones: La Teoría de Orem Déficit de autocuidado y sus postulados, es decir, sus tres teorías relacionadas entre sí: Autocuidado, Teoría del Déficit de Autocuidado y la teoría de sistemas, que abarcan numerosos conceptos, epistemológico, que puede se aplica en diferentes grupos y situaciones.

Palabras-claves: Teoría de Enfermería; Autocuidado; Atencíon de Enfermería.

\section{Introdução}

Os conceitos e postulados de Dorothea Orem são de grande relevância para a construção do conhecimento no campo das bases teóricas e filosóficas da enfermagem, como forte norteador da prática da mesma, no que se refere às necessidades de autocuidado. ${ }^{1}$

Atualmente na realidade brasileira, o modelo conceitual de Dorothea Orem é um dos mais pesquisados, fato este justificado a partir de dois aspectos principais: a promoção de saúde por meio de intervenções educacionais do enfermeiro, que realça ao indivíduo e/ou grupos populacionais o autocuidado e aos tratamentos em longo prazo relacionados ao avanço de condições crônicas de doença e as necessidades de cuidados particulares, como em domicílio dentre outras tecnologias, como formas de cuidar de si. ${ }^{2,3}$ 
Orem desenvolveu sua teoria baseada na necessidade de reformular os currículos da enfermagem, pois acreditava que essa tarefa era necessária para a identificação do domínio e limites da enfermagem como uma ciência e arte. O desenvolvimento da Teoria do Déficit de Autocuidado foi motivado pela insatisfação e preocupação com a ausência de um grupo específico sobre conhecimentos de enfermagem, até então inexistente. ${ }^{4,5}$

Dorothea Elizabeth Orem apresenta sua teoria geral de enfermagem, que se compõe de três teorias inter-relacionadas: (1) Teoria do Autocuidado; (2) Teoria do Déficit de Autocuidado; e (3) Teoria de Sistemas de Enfermagem. ${ }^{6}$

A Teoria do Autocuidado alia-se à prática de cuidados executados pelo indivíduo passível de necessidades para manter-se com vida, saúde e bem-estar. Essa teoria engloba: o autocuidado, a atividade de autocuidado, a exigência terapêutica de autocuidado, e os requisitos para o autocuidado. ${ }^{7}$

A Teoria do Déficit do Autocuidado descreve e explica porque razão as pessoas podem ser ajudadas através da enfermagem e a Teoria dos Sistemas descreve e explica as relações que tem de ser criadas e mantidas para que se produza enfermagem. ${ }^{6}$

Diante do exposto tem-se como objetivo descrever o cenário da aplicabilidade da Teoria do Déficit de Autocuidado de Orem no âmbito da enfermagem brasileira.

Esta revisão integrativa justifica-se pela relevância da síntese de novos conhecimentos no âmbito Teoria do Déficit do Autocuidado de Orem em sua amplitude conceitual, epistemológica e prescritiva, corroborando assim para que o enfermeiro possua habilidades técnicas e conhecimentos empíricos necessários para identificar requisitos de autocuidado terapêutico, nos sujeitos em situação deficitária destes, além de otimizar a assistência de enfermagem de forma sistemática.

\section{Metodologia}

Trata-se de uma revisão integrativa, definida como uma pesquisa que sintetizam achados sobre um determinado fenômeno investigado em estudos primários e que teve 
significante penetração na área de enfermagem nas últimas décadas. A realização deste estudo foi norteada pelas seguintes etapas: elaboração do questionamento norteador, estabelecimento dos objetivos da revisão, critérios de inclusão e exclusão dos artigos; definição da temática relevante e que será extraída das pesquisas; seleção dos artigos na literatura; análise dos resultados; discussão dos achados e apresentação da revisão. ${ }^{8,9}$

Destarte, no que se refere aos objetivos deste estudo, foi traçado o seguinte questionamento norteador: qual o cenário de aplicação da Teoria do Autocuidado de Orem na assistência em enfermagem, no Brasil?

Para a busca das produções científicas indexadas nas bases de dados da Literatura Latino-Americana e do Caribe em Ciências da Saúde (LILACS) e Base de Dados Nacionais da Enfermagem (BDEFN) e na Biblioteca Eletrônica, Scientific Eltronic Libray Online (SciELO). Utilizou-se os descritores em saúde: "Teorias de Enfermagem", "Autocuidado" e "Cuidados de Enfermagem", aplicados simultaneamente com a utilização do operador boleano " $A N D^{\prime}$.

Para o refinamento apropriado dos estudos encontrados, utilizaram-se os seguintes critérios de inclusão para a compilação bibliográfica: temática referente à aplicabilidade da Teoria do Déficit do Autocuidado de Orem; produções bibliográficas compreendidas entre os anos de 2009 e 2014; textos gratuitos, completos e disponíveis, online, idioma de publicação em português, inglês e espanhol; aplicabilidade no Brasil. Foram excluídos artigos publicados em outros idiomas, que abordassem outras teorias de enfermagem, estudos em formato de editorial, carta ao editor ou revisão de literatura e não compreendidos entre os anos de 2009 a 2014.

Conforme o processo de seleção dos artigos (Figura 1) foram obtidas para a análise 12 produções bibliográficas entre artigos originais, revisões integrativas, relatos de experiência, e outros, nas Bases de Dados LILACS, BDENF e na Biblioteca virtual Scielo, entre os meses de outubro e novembro de 2014. 


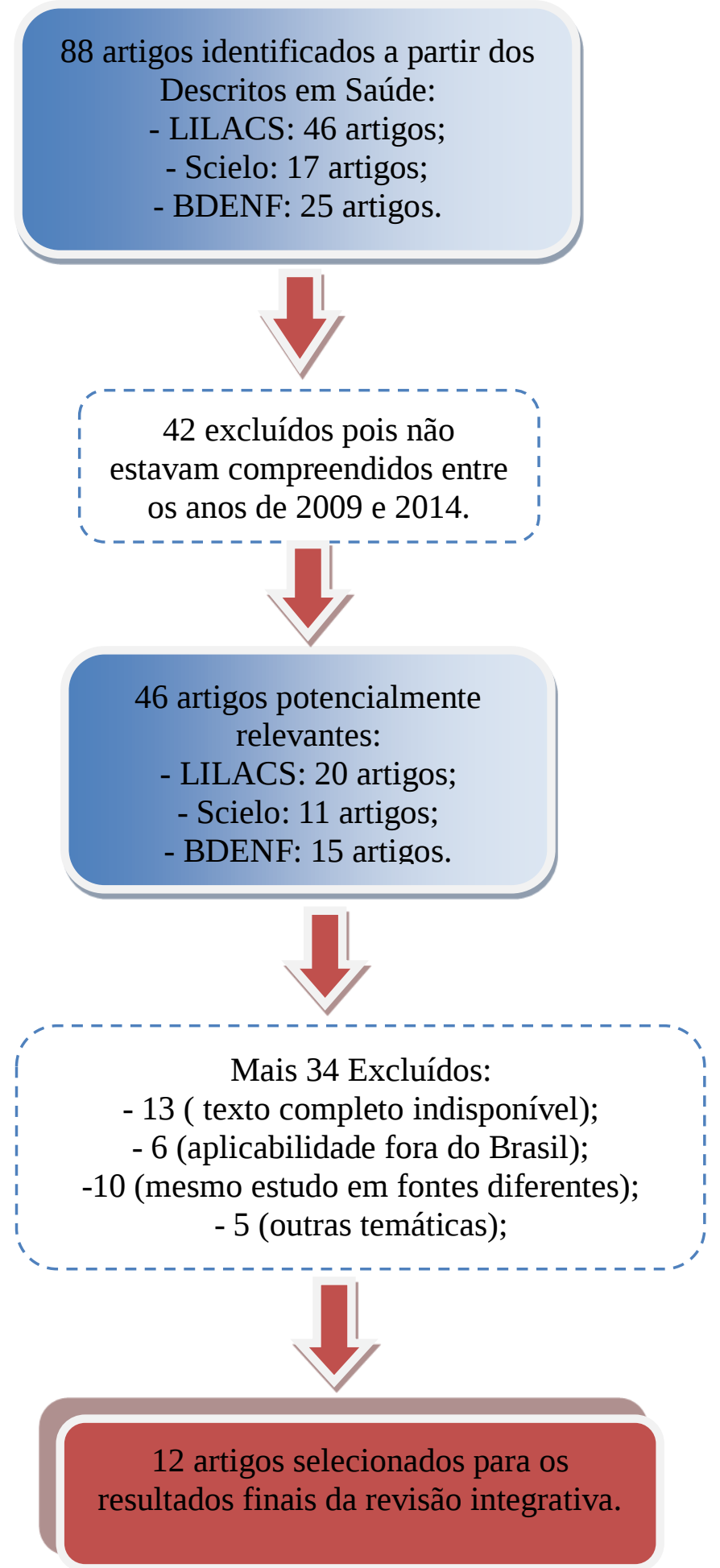

Figura 1: Fluxograma do processo de seleção das produções científicas Brasília-DF, Brasil, 2016.

$$
(\mathrm{N}=12)
$$

Fonte: Elaboração própria (2016).

Inicialmente foram selecionados 88 artigos, com posterior exclusão de 42 artigos, por 
não atenderem ao critério de inclusão - produções científicas compreendidas entre os anos de 2009 e 2014 - resultando em 46 artigos potencialmente relevantes, que após novo refinamento, resultou em mais 34 exclusões (13 textos completos indisponível; 6 não foram aplicados no Brasil; 15 mesmo estudo em bases diferentes; 5 discordantes com a temática: Teoria do Déficit do Autocuidado de Orem), até que se obtivesse o total supracitado de artigos para análise e redação da revisão integrativa.

As produções foram organizados individualmente em quadros e caracterizadas para agrupamento posterior conforme o tipo de estudo, periódico/estado, área da enfermagem (enfermagem, enfermagem médico-cirúrgico, enfermagem psiquiátrica, enfermagem das doenças contagiosas e enfermagem em saúde pública, possibilitando assim que três categorias para análise emergissem: Sistematização da Assistência de Enfermagem, Aplicabilidade da teoria e Educação em saúde para posterior análise e corroboração com a literatura pertinente.

\section{Resultado}

A partir dos descritores em saúde "Teoria de Enfermagem", "Autocuidado" e "Cuidados de Enfermagem", seguindo os critérios de inclusão e exclusão para seleção e refinamentos das produções científicas, foram compiladas um total de 12 destas nas referidas bases de dados, entre os anos de 2009 a 2014 conforme descrito no Quadro 1: 


\begin{tabular}{|c|c|c|}
\hline Título & Autores & Ano \\
\hline $\begin{array}{l}\text { Prática do autocuidado em prostitutas: aplicação } \\
\text { do processo de enfermagem segundo a Teoria de } \\
\text { Orem. }\end{array}$ & $\begin{array}{c}\text { Barbosa IM, Coelho CF, Aquino PS, } \\
\text { Pinheiro ANKB. }\end{array}$ & 2012 \\
\hline $\begin{array}{l}\text { Utilidade da teoria de autocuidado na assistência } \\
\text { ao portador do Vírus da Imunodeficiência } \\
\text { Humana/ Síndrome da Imunodeficiência } \\
\text { Adquirida. }\end{array}$ & $\begin{array}{l}\text { Barroso LMM, Brito DMS, Galvão MTG, } \\
\text { Lopes MVO. }\end{array}$ & 2010 \\
\hline $\begin{array}{l}\text { Autocuidado e recursos assistenciais aos } \\
\text { portadores de hepatite } b \text {. }\end{array}$ & Bento DG, Mariano AF, Andrade SR. & 2010 \\
\hline $\begin{array}{l}\text { Capacidade de autocuidado de adultos e idosos } \\
\text { hospitalizados: implicações para o cuidado de } \\
\text { enfermagem. }\end{array}$ & Costa SRD, Castro, EAB, Acioli S. & 2013 \\
\hline $\begin{array}{l}\text { O convívio do adolescente com HIV/AIDS e o } \\
\text { autocuidado: estudo descritivo }\end{array}$ & $\begin{array}{c}\text { Filho FJS, Gubert FA, Pinheiro PNC, } \\
\text { Martins AKL, Vieira NFC, Nóbrega MFB }\end{array}$ & 2013 \\
\hline $\begin{array}{l}\text { Consulta de enfermagem aplicada a clientes } \\
\text { portadores de hipertensão arterial: uso da teoria } \\
\text { do autocuidado de Orem. }\end{array}$ & Manzini FC; Simonetti JP. & 2009 \\
\hline $\begin{array}{l}\text { Desafios para a introdução da CIPE® no ensino } \\
\text { de Saúde Coletiva: Relato de Experiência. }\end{array}$ & Oliveira MDS, Rocha BS, Bachion MM. & 2013 \\
\hline $\begin{array}{l}\text { Revisão integrativa: etapa inicial do processo } \\
\text { de validação de diagnóstico de enfermagem }\end{array}$ & Pompeo DA, Rossi LA, Galvão CM. & 2009 \\
\hline $\begin{array}{l}\text { Cuidado de Enfermagem segundo a Teoria de } \\
\text { Orem: assistência a paciente com transtorno } \\
\text { afetivo bipolar }\end{array}$ & $\begin{array}{l}\text { Sales DS, Oliveira EN, Brito MCC, } \\
\text { Rodrigues TB, Souza AMM. }\end{array}$ & 2013 \\
\hline $\begin{array}{l}\text { Necessidades de orientação de enfermagem } \\
\text { para o autocuidado de clientes em terapia de } \\
\text { hemodiálise }\end{array}$ & Santos I, Rocha RPF, Berardinelli LMM & 2011 \\
\hline $\begin{array}{l}\text { Realização do auto-exame das mamas por } \\
\text { profissionais de enfermagem }\end{array}$ & $\begin{array}{l}\text { Silva RM, Sanches MB, Ribeiro NLR, } \\
\text { Cunha FMAM, Rodrigues MSP. }\end{array}$ & 2009 \\
\hline $\begin{array}{l}\text { Self care in neurogenic intestine in subjects } \\
\text { with spinal cord injury: an integrative review }\end{array}$ & $\begin{array}{c}\text { Vasconcelos AS, França ISX, Coura AS, } \\
\text { Enders BC , Cartaxo HGO, Sousa FS. }\end{array}$ & 2013 \\
\hline
\end{tabular}

Quadro 1. Caracterização das produções científicas evidenciadas a partir do título, autores e ano. Brasília-DF, Brasil, 2016. ( $\mathrm{N}=12)$.

Fonte: Elaboração própria (2016).

No que concerne a produtividade de pesquisas relacionadas à aplicabilidade da Teoria do Déficit de Autocuidado de Orem no âmbito nacional, observou-se que o ano de 
maior produtividade, conforme os critérios previamente estabelecidos, foi o ano de 2013 $(41,6 \%)$.

Quanto a caracterização da bibliografia selecionada neste estudo, optou-se pela abordagem dos itens tipo de estudo, periódico, área da enfermagem (classificação CAPES) e população, conforme a situação de saúde-doença dos mesmos.

No que se refere ao tipo de estudo (Tabela 1), evidenciou-se que os estudos do tipo descritivo com abordagem qualitativa e descritivo com abordagem qualitativa foram os mais utilizados como método nos achados, ambos com frequência absoluta de 4 e relativa de 33\%. Também foram utilizadas as tipologias, revisão integrativa/bibliográfica (2; 17\%), relato de experiência e metodológico e de tecnologia assistencial, que obtiveram frequência de 1 (8,3\%) por produção bibliográfica. Quanto aos periódicos, identificou-se que os mais utilizados para as publicações foram a Enfermagem em Foco, Acta Paulista Enfermagem e OBJN, que apresentaram igual frequência absoluta e relativa de ocorrência na investigação $(2 ; 17 \%)$.

Tabela 1. Distribuição do tipo de estudo e periódico das produções selecionadas. Brasil. 2009-2014. Brasília-DF, 2016

\begin{tabular}{lccc}
\multicolumn{1}{c}{ Tipo de estudo } & Periódico/estado & $\mathbf{n}$ & 1 \\
& OBJN & 1 \\
& Enfermagem em foco & 1 & 33,3 \\
Descritivo/qualitativo & Cienc Cuid Saude & 1 & 1 \\
& Acta Paul Enferm & 1 & 33,3 \\
& Rev Esc Enferm USP & 1 \\
\hline Descritivo/quantitativo & Rev Latino-am Enfermagem & 1 & 1 \\
\hline Revisão Integrativa/bibliográfica & Rev Min Enferm & OBJN & 1 \\
\hline Relato de experiência & Acta Paul Enferm & 8,3
\end{tabular}


Total

Fonte: Elaboração própria (2016).

Tabela 2. Distribuição em relação à área da enfermagem e população das produções selecionadas. Brasil. 2009-2014. Brasília-DF, 2016.

\begin{tabular}{|c|c|c|c|}
\hline Área da enfermagem & População & $\mathbf{n}$ & $\%$ \\
\hline $\begin{array}{l}\text { Enfermagem } \\
\text { psiquiátrica }\end{array}$ & $\begin{array}{l}\text { Idosa com Transtorno de } \\
\text { Afetividade Bipolar }\end{array}$ & 1 & 8,3 \\
\hline $\begin{array}{l}\text { Enfermagem em } \\
\text { doenças infecciosas }\end{array}$ & $\begin{array}{c}\text { Adolescentes/adultos com } \\
\text { HIV/AIDS }\end{array}$ & 3 & 25 \\
\hline \multirow{6}{*}{$\begin{array}{l}\text { Enfermagem em Saúde } \\
\text { Pública }\end{array}$} & $\begin{array}{c}\text { Indivíduos com Hipertensão } \\
\text { Arterial Sistêmica }\end{array}$ & 2 & 17 \\
\hline & $\begin{array}{c}\text { Lesão medular e intestino } \\
\text { neurogênico }\end{array}$ & 1 & 8,3 \\
\hline & Prostitutas & 1 & 8,3 \\
\hline & $\begin{array}{l}\text { Adultos e idosos } \\
\text { institucionalizados }\end{array}$ & 1 & 8,3 \\
\hline & Hanseníase e Diabetes Mellitus & 1 & 8,3 \\
\hline & $\begin{array}{c}\text { Profissionais de } \\
\text { enfermagem/autoexame das } \\
\text { mamas }\end{array}$ & 1 & 8,3 \\
\hline Total & & 12 & 100 \\
\hline
\end{tabular}

Fonte: Elaboração própria (2016).

No que concerne às áreas da enfermagem e população mais evidenciadas nos artigos selecionados para este estudo (Tabela 2), destacaram- se a enfermagem das doenças infecciosas, em indivíduos doentes por HIV/AIDS (3; 25\%). Por conseguinte, evidenciou-se que a Enfermagem em Saúde Pública, também foi constantemente abordada no que se refere à indivíduos com Hipertensão Arterial Sistêmica (2; 17\%), Lesão medular e intestino neurogênico, Prostitutas, Adultos e idosos institucionalizados, Hanseníase e Diabetes Mellitus, cada um destes, alcançou as frequências de uma produção e 8,3\%. 
Ademais, através da leitura e releitura dos 12 artigos que formam o corpus de dados desse trabalho, foi possível estabelecer três categorias temáticas: Sistematização da Assistência de Enfermagem, aplicabilidade da teoria e educação em saúde. ${ }^{4}$

\section{Discussão}

Alguns autores contribuem que a Teoria do Autocuidado de Orem, é uma das mais pesquisadas na enfermagem brasileira $(n=23 ; 13,5 \%)$ estando atrás das Teorias de Enfermagem de Paterson e Zaderad ( $n=29 ; 17 \%)$ e a Teoria do Cuidado Cultural de Leineger $(n=27 ; 15,8 \%)^{2}$

Outros corroboram ainda que a Teoria do Autocuidado de Orem foi a mais frequente, no que concerne à referencias em enfermagem utilizados na assistência, entre os anos de 2002 e 2007, no Brasil, com frequência absoluta de 24 e relativa de 14,8\% em um total de 165 artigos analisados em seu estudo bibliográfico. ${ }^{3}$

A tendência metodológica comumente utilizada em demais estudos de enfermagem, a abordagem qualitativa (154; 58,5\%) na gama de produtividade acadêmica monográfica de estudantes de enfermagem de uma faculdade do norte mineiro. ${ }^{10}$

A maior ocorrência de estudos relacionados a doenças infectocontagiosas como HIV/AIDS divergem de outros estudos que demonstraram que entre os anos de 2005 a 2008 a maioria da produtividade acadêmica em enfermagem, em nível de mestrado, abordou a temática enfermeiros e equipe de enfermagem. ${ }^{11}$

Seguem-se a análise das categorias que emergiram das produções científicas evidenciadas: Sistematização da Assistência de Enfermagem -SAE, Aplicabilidade da Teoria e Educação em Saúde.

\section{Sistematização da Assistência de Enfermagem - SAE}

A aplicação do processo de enfermagem, a partir da Teoria do Déficit do Autocuidado de Orem, traz muitos benefícios às ações do cuidar para a melhoria na qualidade de vida do 
paciente. Orem apresenta um método de determinação das deficiências de autocuidado e a posterior definição dos papéis da pessoa ou enfermeiro para satisfazer as exigências de autocuidado. $^{12}$

As condições crônicas de doenças têm exigido dos indivíduos a utilização de tratamento a longo prazo, o uso de tecnologias de cuidado no domicílio e a reestruturação de estilos de vida como formas de cuidado de si, é uma confirmação de que a Teoria de Orem é importante para a prática da enfermagem. ${ }^{13}$

Nesta ótica, se enfatiza a segunda etapa da aplicação do Processo de Enfermagem, norteado pela Teoria do Autocuidado de Orem, ou seja, pelos diagnósticos de enfermagem, para indivíduos diagnosticados e em tratamento para HIV/AIDS, com requisitos de autocuidado permanentes e que necessitam da promoção do autocuidado terapêutico, contribuindo assim para que as intervenções de enfermagem sejam adequadas e direcionadas às necessidades existentes destes indivíduos. Outros estudos, realizados com indivíduos em tratamento para Hipertensão Arterial Sistêmica, também destacam a relevância da aplicação do Processo de Enfermagem em sua completude, como a própria consulta de enfermagem em sua essência técnico-científica, a partir das noções de autocuidado, também norteada pelo Conceitual de Orem. ${ }^{14,15}$

Destaca-se a relevância dos diagnósticos de enfermagem listados e norteados a partir da Teoria do Autocuidado de Orem, como contribuinte para a elaboração das intervenções de enfermagem aos diagnósticos de enfermagem, a saber: Risco de constipação, constipação e incontinência intestinal, para indivíduos com intestino neurogênico e lesão raquimedular. ${ }^{16}$

A Teoria do Autocuidado leva a uma reflexão de como somos, de como e porque nos cuidamos e porque quando não conseguimos nos cuidar necessitamos dos outros, sendo considerado como algo inerente à humanidade. Essa teoria norteia o desenvolvimento de práticas científicas e de reflexões sobre a sociedade, de forma coerente com as exigências de cuidado sentidas por cada indivíduo ou grupo. ${ }^{3}$ 
Estudos relacionados à aplicabilidade da $\mathrm{SAE}$, baseada no modelo de Orem, por alunos de graduação de enfermagem em unidades de campo de prática contribui para a qualidade da assistência, para melhor estruturação do planejamento de ações com base em evidências científicas, para maior segurança e para a definitiva implantação do processo de enfermagem nas instituições de saúde. ${ }^{13}$

A enfermagem como disciplina é influenciada pela ação de forças de diferentes características, sejam sociais, culturais, epistemológicas, interligando-se com outros saberes prevalecentes em cada época, as teorias de enfermagem são produtos da reflexão dos profissionais no interior da disciplina, mas também nas discussões e intersecções com o conhecimento científico em geral. As reflexões sobre os modos de fazer na profissão constituem possibilidades para aperfeiçoar e amadurecer as teorias existentes em enfermagem, não só valorizando sua concepção teórica, mas também sua utilidade prática no exercício profissional, no cuidado, seja ele orientado para o indivíduo ou a comunidade. ${ }^{3}$

O uso de teorias de enfermagem na aplicação da Sistematização da Assistência de Enfermagem permite a explicitação dos referenciais teóricos que justificam as diferentes práticas da profissão, e assim, a construção de projetos coletivos e discursos próprios da disciplina de enfermagem.

\section{Aplicabilidade da Teoria}

Em um estudo realizado em um grupo de prostitutas evidenciou-se uma maior facilidade na abordagem do indivíduo, contribuindo assim para a interação e a detecção das alterações nos requisitos de autocuidado. Ademais, a elaboração do plano de cuidados em conformidade com as pacientes permitiu maior adesão às orientações e proporcionou uma valorização das necessidades prioritárias. ${ }^{17}$

A aplicabilidade da teoria de Dorothea Orem pode ser expandida a diversos indivíduos e grupos, favorecendo uma prática de enfermagem pertinente às necessidades de autocuidado do indivíduo e/ou grupo. 
Evidências bibliográficas corroboram que utilidade da Teoria do Autocuidado de Orem para indivíduos portadores do Vírus da Imunodeficiência Adquirida - AIDS demonstrou ser útil para orientar a SAE ao usuário em tratamento para HIV/AIDS, além de contribuir para a construção de instrumentos de avaliação e orientação, bem como possibilitar a mensuração da qualidade da assistência; testar modelos; caracterizar de forma holística e conceitual o paciente e reforçar o papel educativo do enfermeiro na díade profissional-paciente. $^{18}$

Outro estudo utilizou a teoria de Orem para classificar e avaliar adultos e idosos hospitalizados para o tratamento clínico de patologias ou agravos crônicos quanto a capacidade de autocuidado, identificando a diferença entre os gêneros e evidenciando o grupo com grau de dependência que necessita da presença de um cuidador familiar. Os autores afirmam que, considerando os sistemas de enfermagem propostos por Dorothea Orem, tanto o adulto ou o idoso hospitalizado quanto o seu cuidador familiar precisam passar pelo processo de apoio-educação do profissional enfermeiro para que possam dar continuidade ao autocuidado após a alta hospitalar. ${ }^{19}$

Sobre a aplicação do sistema apoio-educação a partir das intervenções baseadas no sistema apoio educação, pôde-se contribuir para o desenvolvimento da capacidade de execução do autocuidado, com conhecimento e maior segurança. Porém, os autores ressaltam que o sucesso das intervenções se deve ao interesse e à capacidade do indivíduo em seu cuidado. ${ }^{17}$

No sistema apoio-educação, o indivíduo consegue executar o autocuidado, assim suas exigências resumem-se na tomada de decisões, controle do comportamento e aquisição de conhecimentos e habilidades. O enfermeiro como o educador, promove ao cliente, a partir também da demanda deste, a capacidade dele se tornar um agente de autocuidado. $^{19}$

Um grupo de autores trouxe como conclusão de sua pesquisa o conhecimento produzido aproxima a aplicação da Teoria do Autocuidado aos desvios de saúde de natureza 
sexualmente transmissível. Neste sentido, traz contribuições ao entendimento da possibilidade de cronificação de doenças transmissíveis e à responsabilidade dos profissionais de saúde por realizar, em conjunto com os portadores da patologia, os requisitos do autocuidado. ${ }^{20}$

\section{Educação em saúde}

Com a mudança do perfil demográfico e epidemiológico da população brasileira, é importante a realização de educação em saúde, que deve ser vista como uma dimensão do processo de cuidar pelo enfermeiro, sobretudo pela convivência direta com a população, tanto no ambiente hospitalar quanto na comunidade, incluindo a orientação quanto aos comportamentos de risco, de forma a promover a saúde e prevenir as doenças crônicas. ${ }^{17}$

O enfermeiro deve agir de forma a orientar sobre os cuidados, tanto aos pacientes quanto aos cuidadores familiares, visando minimizar o grau de dependência que tende a aumentar a morbimortalidade da população.

Os programas de preparo para a alta hospitalar podem ser usados como uma estratégia de educação em saúde, e devem ser incentivados, contribuindo para a integralidade do cuidado, a fim de que a pessoa dependente receba um tratamento resolutivo e humanizado, reduzindo a possibilidade de reinternações. ${ }^{17}$

Sobre a educação em saúde alguns autores reforçam em seu estudo que devido a alta probabilidade de disseminação do vírus da hepatite, são necessárias medidas de promoção e prevenção em saúde, bem como o comprometimento com o autocuidado, não apenas do portador, mas também de sua família, de seu círculo social e, fundamentalmente, dos responsáveis pela oferta de serviços de saúde que sejam acessíveis e atendam ao princípio da integralidade. ${ }^{20}$

Utilizando-se da tipologia metodológica de tecnologia assistencial, com o objetivo de construir um protocolo de assistência de enfermagem para o paciente em pré e pósoperatório de cirurgia bariátrica, discorre-se que esta tecnologia é um importante recurso para instrumentalizar as orientações prestadas pelos enfermeiros ao paciente bariátrico, 
visando ao autocuidado, além de dar subsídios para sistematizar a assistência de enfermagem a ser prestada, otimizando assim a assistência em enfermagem.

A Teoria de Orem tem sido utilizada no Brasil para ações educativas realizadas por enfermeiros, no desenvolvimento de atitudes que facultem aos indivíduos e/ou grupos populacionais o autocuidado. ${ }^{13}$

A abordagem qualitativa em relação ao enfrentamento de adolescentes que convivem com o Vírus da Imunodeficiência Humana - HIV por meio de ações de autocuidado, destacou o papel educativo do enfermeiro, a partir da fala dos próprios sujeitos do estudo, no que se refere ao compartilhamento e participação do processo apoioeducação para o conhecimento dos requisitos de autocuidado terapêutico, bem como exercício do mesmo.

Afirma-se que cabe à enfermagem orientar a pessoa na sua adaptação como o meio ambiente. Já o cuidado de si, segue os pressupostos do paradigma da simultaneidade que valoriza o subjetivo do ser humano compreendendo que a enfermagem deve auxiliá-lo, respeitando a sua vivência, nos cuidados a sua saúde, tendo como meta uma melhoria da sua qualidade de vida. ${ }^{10}$

Deve-se salientar, ainda, que a educação em saúde por meio de práticas educativas precisa ser desenvolvida em todos os âmbitos de atenção à saúde de forma articulada, seja na atenção terciária, por meio da preparação do paciente e familiar durante toda a internação para o momento da alta; seja na atenção secundária, pelas consultas ambulatoriais, onde os profissionais de saúde e, em especial o enfermeiro, devem aproveitar a oportunidade para ensinar e esclarecer dúvidas por meio de consultas de enfermagem; e na atenção primária, por meio de visitas domiciliares, aproveitando a Estratégia em Saúde da Família (ESF) a fim de monitorar os cuidados realizados. ${ }^{19}$

No âmbito da enfermagem psiquiátrica, evidenciou-se que a implementação da Sistematização da Assistência em Enfermagem à luz do conceitual de Dorothea Orem, evidenciou, juntamente com os seus colaboradores, o quanto que o Processo de 
Enfermagem facilitou a comunicação educativa ao estudo de caso aplicado à um idosa portadora de Transtorno Afetivo Bipolar, contribuindo para o alcance de muitas metas estabelecidas no plano assistencial de enfermagem. ${ }^{21,22}$

Percebe-se que o cuidado é uma característica do ser humano, adotada pela enfermagem, tornando-se sua essência - o cuidado de enfermagem. Por sua vez, o cuidado de enfermagem, quando é executado pelo outro, para manter seu bem-estar, é descrito por dois termos: o autocuidado e o cuidado de si, que não possuem somente uma diferença semântica, mas sim paradigmática. ${ }^{19}$

\section{Conclusões}

A Teoria do Déficit do Autocuidado de Orem, bem como seus postulados, ou seja, suas três teorias inter-relacionadas: Teoria do Autocuidado, Teoria do Déficit do Autocuidado e Teoria de Sistemas, abrangem inúmeros conceitos, de natureza epistemológica, que podem ser aplicados em diferentes grupos e situações.

O autocuidado, seu déficit e os requisitos para o mesmo são os conceitos básicos mais norteadores para a escolha da Teoria Geral do Autocuidado, embora, deste ponto deva-se considerar o referencial teórico das outras disciplinas como ciências sociais e comportamentais que contribuíram também para a compilação das principais definições que fundamentam e fortalecem teoricamente, de forma empírica, o conceitual de Dorothea Orem.

Ratifica-se a importância do conhecimento filosófico para o entendimento conceitual das teorias de enfermagem, pois a prática sem a devida fundamentação científica pode trazer lacunas e divergências conceituais que influenciarão a eficácia da assistência, tão somente naquelas circunstâncias que requerem intervenções que promovam o autocuidado, mas em toda e qualquer definição teórica que dê fundamentos para a assistência de enfermagem. 


\section{Referências}

1. Santos I, Rocha RPF, Berardinelli LMM. Necessidades de orientação de enfermagem para o autocuidado de clientes em terapia de hemodiálise. Rev Bras Enferm. 2011marabr;64(2):335-42. http://dx.doi.org/10.1590/S0034-71672011000200018.

2. Schaurich D, Crossetti MGO. Produção do conhecimento sobre teorias de enfermagem: análise de periódicos da área, 1998-2007. Esc Anna Nery. 2010;14(1):182-88. http://dx.doi.org/10.1590/S1414-81452010000100027.

3. Rosa LM, Sebold LF, Arzuaga MA, Santos VEP, Radüz V. Referenciais de enfermagem e produção do conhecimento científico. Rev Enferm UERJ [Internet]. 2010 [acesso em 03 maio 2016];18(1):120-5. Disponível em: http://www.facenf.uerj.br/v18n1/v18n1a21.pdf.

4. Fawcett, J. As Teoristas de Enfermagem: 21st-CenturyUpdates-Dorothea E. Orem. Revista Trimestral de Ciências da Enfermagem, v. 14 ,n 1, janeiro, 34-38, 2001.

5. McEwen M, Wills EM. Bases Teóricas para Enfermagem. Porto Alegre: Artmed; 2009. 576 p.

6. Orem DE, Taylor SG, Renpenning KM. (col.) Nursing: concepts of practice. 6th ed. St. Louis: Mosby. 2001. 542 p.

7. Leopardi MT. Teoria e método em assistência de enfermagem. Florianópolis: Ed. Soldasoft, 2006.

8. Soares CB, Hoga LAK, Peduzzi M, Sangaleti C, Yonekura T, Silva DRAD. Revisão integrativa: conceitos e métodos utilizados na enfermagem. Rev Esc Enferm USP. 2014;48(2):335-45. https://doi.org/10.1590/S0080-6234201400002000020.

9. Souza MT, Silva MD, Carvalho R. Revisão integrativa: o que é e como fazer. Einstein [Internet]. 2010 [acesso em 24 nov 2014];8(1 Pt 1):102-6. Disponível em: http://apps.einstein.br/revista/arquivos/PDF/1134-Einsteinv8n1_p102-106_port.pdf

10. Silva, RM, Sanches MB, Ribeiro NLR, Cunha FAM, Rodrigues MSP. Realização do autoexame de mamas por profissionais de enfermagem. Rev Esc Enferm USP. 2009;43(4):902-8. http://dx.doi.org/10.1590/S0080-62342009000400023.

11. Prado ML, Rocha PK, Bakes VMS, Reibinitz KS, Waterkemper R, Gomes, DC. Produção de conhecimento em um curso de mestrado em enfermagem no Brasil. Cincia Enferm. 2011;2(3):43-50. http://dx.doi.org/10.4067/S0717-95532011000300005. 
12. Bezerra, MLR, Ribeiro PRS, Sousa AA, Costa AIS, Batista TS. Diagnósticos de enfermagem conforme a teoria do autocuidado de Orem para pacientes em tratamento hemodialítico. Rev Cienc Ext [Internet]. 2012 [acesso em 20 nov 2014];8(1):60-81.

13. Oliveira MDS, Rocha BS, Bachion MM. Desafios para a introdução da CIPE $®$ no ensino de Saúde Coletiva: relato de experiência. Enferm Foco. 2013;4(1):7-10. https://doi.org/10.21675/2357-707X.2013.v4.n1.493.

14. Cunha GH, Galvão MTG. Diagnósticos de enfermagem em pacientes com o vírus da imunodeficiência humana: síndrome da imunodeficiência adquirida em assistência ambulatorial. Acta Paul Enferm. 2010;23(4):526-32. http://dx.doi.org/10.1590/S010321002010000400013.

15. Manzini FC, Simonetti JP. Consulta de enfermagem aplicada a clientes portadores de hipertensão arterial: uso da teoria do autocuidado de Orem. Rev Latino-Am Enferm. 2009;17(1):113-9. http://dx.doi.org/10.1590/S0104-11692009000100018.

16. Vasconcelos AS, França ISX, Coura AS, Enders BC, Cartaxo HGO, Sousa, FS. Self care in neurogenic intestine in subjects with spinal cord injury: an integrative review. O Braz J Nurs. 2013;12(4): 998-1010. https://doi.org/10.5935/1676-4285.20133692.

17. Barbosa IM, Coelho CF, Aquino PS, Pinheiro AKB. Prática do autocuidado em prostitutas: aplicação do processo de enfermagem segundo a teoria de Orem. Enferm Foco. 2012;3(1):36-41. https://doi.org/10.21675/2357-707X.2012.v3.n1.218.

18. Barroso LMM, Brito DMS, Galvão MTG, Lopes MVO. Utilidade da teoria de autocuidado na assistência ao portador do Vírus da Imunodeficiência Humana/ Síndrome da Imunodeficiência Adquirida. Acta Paul Enferm. 2010;23(4):562-7. http://dx.doi.org/10.1590/S0103-21002010000400019.

19. Costa SRD, Castro EAB, Acioli, S. Capacidade de autocuidado de adultos e idosos hospitalizados: implicações para o cuidado de enfermagem. Rev Minei Enferm. 2013;17(1):192-9. http://www.dx.doi.org/10.5935/1415-2762.20130016.

20. Bento DG, Mariano AF, Andrade SR. Autocuidado e recursos assistenciais aos portadores de hepatite B. Cienc Cuid Saude. 2010;9(2):285-91. http://dx.doi.org/10.4025/cienccuidsaude.v9i2.8526.

21. Félix LG, Soares MJGO, Nóbrega MML. Protocolo de assistência de enfermagem ao paciente em pré e pós-operatório de cirurgia bariátrica. Rev Bras Enferm. 2012;65(1):8391. http://dx.doi.org/10.1590/S0034-71672012000100012. 
22. Sales DS, Oliveira EN, Brito MCC, Rodrigues TB, Sousa AMA. Cuidado de enfermagem Segundo a Teoria de Orem: assistência a paciente com transtorno afetivo bipolar. J Res Fundam Care. 2013;5(3):311-7. http://doi.org/10.9789/2175-5361.2013v5n3p311.

\section{Minicurrículo}

Maria Luiza Rêgo Bezerra | ORCiD: 0000-0002-3336-7760

Mestra em Enfermagem pelo Programa de Pós-graduação em Enfermagem da Universidade de Brasília - UnB. Especialista em Gestão em Saúde Pública com Ênfase em Saúde Coletiva e da Família (INESPOPUC/SP). Enfermeira pela Universidade Federal do Maranhão - UFMA, campus de Imperatriz. Atualmente é Oficial da Marinha do Brasil atuando no Departamento de Enfermagem, área assistencial, no Centro Cirúrgico do Hospital Naval de Brasília, DF.

Renata de Paula Rocha Faria | ORCiD: 0000-0002-5932-7441

Doutora em Enfermagem pela Universidade de Brasília - UnB.

Cristine Alves Costa de Jesus | ORCiD: 0000-0002-8638-4468

Doutorado em Enfermagem Fundamental pela Escola de Enfermagem de Ribeirão Preto da Universidade de São Paulo - USP. Líder do Grupo Fundamentos e Tecnologias do Cuidar em Enfermagem, vinculado ao CNPq. Professora Associada do Departamento de Enfermagem da Faculdade de Ciências da Saúde da UnB. Docente do Programa de Pós-Graduação em Enfermagem da UnB. É membro da NANDA-I (North American Nursing Diagnosis Association International) e da Sigma Theta Tau.

Paula Elaine Diniz dos Reis | ORCiD: 0000-0002-9782-3366

Doutorado Direto pelo Programa Enfermagem Fundamental pela Escola de Enfermagem de Ribeirão Preto da Universidade de São Paulo - USP (2008). Pós-Doutorado pela University of Washington, Seattle, USA (2016).

Diana Lúcia Moura Pinho | ORCiD: 0000-0003-4212-2340

Graduação em Enfermagem pela Universidade Católica do Salvador. Mestra em Educação pela Universidade de Brasília - UnB. Doutora em Psicologia pela UnB. Professora Associada da UnB/Faculdade UnB Ceilândia e do Departamento de Enfermagem da Faculdade de Ciências da Saúde. Professora Visitante Honorária da Faculty of Health, Social Care \& Education, Kingston \& St Georges University of London.

Ivone Kamada | ORCiD: 0000-0003-2569-8727

Doutorado em Enfermagem pela Universidade de São Paulo - USP. Professora Associado da Universidade de Brasília - UnB. Membro da Comissão do Programa de Pós-Graduação em Enfermagem da UnB. Credenciada pelo Programa de Pós-Graduação em Enfermagem da UnB (mestrado e doutorado). Consultor ad hoc área da Enfermagem da CAPES. É consultora ad hoc da Revista da Escola de Enfermagem da USP - REEUSP, Revista Texto e Contexto em Enfermagem, Revista Brasileira de Enfermagem, Revista Estima. 